\title{
Diseño de una Herramienta de Evaluación de la Seguridad en Grandes Pabellones Deportivos respecto a los Riesgos Individuales y Colectivos: Aplicación en un Estudio de Caso
}

\section{Development of a Tool for the Assessment of the Safety in Sports Arenas in respect of Individual and Collective Risks: Application to a Case Study}

\author{
María José Maciá Andreu*, Ana María Gallardo Guerrero, Javier Sánchez Sánchez y José Luis Gómez-Calvo
}

Universidad Católica San Antonio de Murcia

\begin{abstract}
Resumen: Los gestores deportivos, organizadores de eventos y titulares de las instalaciones, son los responsables de proporcionar unas condiciones óptimas de seguridad a usuarios, espectadores y trabajadores de las mismas, a través de una valoración y control de los riesgos que se presentan. Es por ello que el objetivo de este estudio, ha sido elaborar una herramienta de evaluación de la seguridad en grandes pabellones deportivos respecto a los riesgos individuales y colectivos, basada en la legislación actual de ámbito estatal, normas NIDE y UNE-EN, así como manuales de buenas prácticas. Esta lista de control es de respuesta dicotómica (SI/NO) y consta de un total de 218 ítems (147 relacionados con riesgos individuales y 71 respecto a los colectivos) divididos en las 10 áreas que componen la instalación, con el fin de facilitar el análisis y posterior control. Esta herramienta se ha utilizado para la realización de un estudio piloto de la seguridad de un gran pabellón, del cual se extrae que el área con un porcentaje de cumplimiento mayor es la de "Exteriores y Acceso" con un 95\%, situándose el área de "Emergencias" en el extremo opuesto con un 65,5\%. Las deficiencias observadas, que suponen un $17,88 \%$ del total, nos llevan a recomendar la utilización de esta lista de control como método de detección de riesgos que, en la mayoría de los casos, son susceptibles de eliminación o reducción, consiguiendo con ello unas mejoras considerables en la seguridad de la instalación.

Palabras clave: gestión deportiva, lista de control, prevención, instalación deportiva, equipamiento deportivo, gestión de emergencias.
\end{abstract}

Abstract: Sport managers, event holders and owners of sport facilities, are responsible for providing optimum safety conditions for users, spectators and employees, through the assessment and control of the potential risks. That is why the purpose of this study has been the development of a safety analysis tool for big sports arenas with respect to individual and collective risks, based on Spanish regulations, NIDE and UNE-EN standards and best practice manuals. This checklist with dichotomous response (YES/ $\mathrm{NO}$ ), consists of 218 items (147 of them related to individual risks and 71 regarding collectives) divided into 10 areas that compose the facilities, with the aim of enabling risk analysis and its subsequent control. A pilot test was carried out in order to assess the safety in a big sport arena, from which is extracted that the area with the highest level of compliance is "Outdoors and access" with a 95\%, while the area of "Emergencies" stands at the other end of the scale with a $65,5 \%$. The detected weaknesses, representing $17,88 \%$ of total, lead us to recommend the application of this checklist as a method of risk detection that, in most cases, are susceptible to removal or reduction, thereby achieving a considerable improvement in the safety of the facilities.

Key words: sports management, checklist, prevention, sports facility, sports equipment, emergency management.

\section{Introducción}

La seguridad es un derecho de usuarios y trabajadores y, consecuentemente, los titulares de las instalaciones y/o organizadores de actividades deportivas tienen la obligación legal de garantizarla. Su omisión puede suponer la comisión de infracciones que conlleven sanciones administrativas o económicas, además de posibles responsabilidades legales (Gómez-Calvo, 2005, 2014).

Las consecuencias de no atender a los posibles riesgos que se presentan, son que éstos pueden materializarse en accidentes deportivos de diversa índole, siendo la proactividad el primer paso por parte de los responsables de las instalaciones para detectarlos (Hall, Cooper, Marciani y McGee, 2012).

Dirección para correspondencia [Correspodence address]: María José Maciá Andreu. Universidad Católica San Antonio de Murcia. E-mail: mjmandreu@gmail.com
Debido a que muchos de ellos son prevenibles (Parkkari, Kujala y Kannus, 2001), el control de los mismos por parte de las administraciones públicas y entidades privadas, debe garantizarse en todos sus ámbitos, debiendo constituir una prioridad para el gestor deportivo (Finch, Donaldson, Mahoney y Otago, 2009; Fuller, 2007) y para los titulares de la misma (Gómez-Calvo, 2005). Este hecho se hace indispensable teniendo en cuenta que, en el análisis de los principales accidentes y situaciones de emergencia ocurridos durante los últimos años, se arroja que la causa de los mismos fue en un $58 \%$ una insuficiente previsión de las causas, seguido de un $22 \%$ de la falta de equipamientos de emergencia y un $12 \%$ del mal estado de las instalaciones (Shangjun y Xinjian, 2012).

Los centros dedicados a la salud y el fitness, como es el caso de los grandes pabellones deportivos objeto de estudio, son focos de concentración de accidentes (Parkkari et al., 2004; 
Requa, DeAvilla, y Garrick, 1993). Éstos presentan algunos de los principales factores de riesgo que han influido como desencadenantes de grandes desastres previamente, entre los que se encuentran la alta concentración de personas, bajo control de multitudes, insuficiente seguridad contra incendios, carencias en los locales de atención médico sanitaria, fallos en la comunicación entre la organización y el equipo médico, condiciones de temperatura adversas, insuficiencia de salidas de emergencia y deficiencias en los controles de acceso al evento (De Castro, Simóes, Delamarque y Pepe, 2014).

A la hora de seleccionar las referencias para evaluar la seguridad, se atiende a la máxima que enuncia que "Limitarse al cumplimiento de los requisitos, es quedarse en el umbral de las necesidades" (Gómez-Calvo, 2004). De este modo, si bien la legislación es de obligado cumplimiento y su ejecución debe de estar garantizada, existen otras normativas que se hacen necesarias para una adecuada previsión y control de los riesgos que se presentan en la instalación.

Es por todo lo anterior, que los objetivos del presente estudio son, por un lado, elaborar una herramienta útil para el gestor deportivo que permita detectar y analizar los posibles riesgos para la seguridad que presenta el pabellón deportivo, respecto a los riesgos individuales y grandes daños colectivos. Y por otro, aplicar dicha herramienta en un gran pabellón deportivo para comprobar su adecuación al fin para la que ha sido elaborada.

\section{Método}

La investigación llevada a cabo es de tipo no experimental y descriptiva, si bien el trabajo previo de creación de la herramienta se enmarca dentro de la investigación exploratoria. Este estudio descriptivo es de tipo cuantitativo y transversal.

\section{Muestra}

Para valorar la idoneidad de la herramienta, se analizó un gran pabellón deportivo de titularidad municipal con un aforo total de 2.424 personas, en el cual se llevan a cabo actividades deportivas de diversa índole, así como eventos y competiciones de carácter regional, nacional e internacional.

\section{Herramienta de recogida de datos}

Para la realización del estudio, se tomó como referencia la herramienta, así como su proceso de creación, utilizada por López-Fernández et al. (2014), optando de este modo por la elaboración de una lista de control o checklist de respuesta dicotómica (SI/NO) en función del cumplimiento o no del ítem descrito, pretendiendo con ello la obtención de datos de carácter objetivo a través de un procedimiento de auditoría.

Dentro de la complejidad y carácter extenso que supone la valoración de la seguridad a través de los posibles riesgos que se presentan, Gómez-Calvo (2013) establece un "catálogo de riesgos” en el cual se basó el desarrollo de la herramienta (tabla 1):

Tabla 1. Catálogo de riesgos (Gómez-Calvo, 2013).

\begin{tabular}{l}
\hline Catálogo de riesgos \\
\hline Riesgos de dańos por actos deliberados o incívicos. \\
Riesgos de graves daños colectivos. \\
Riesgos de daños individuales. \\
Riesgos para los derechos de los usuarios. \\
Riesgos laborales. \\
\hline
\end{tabular}

Dentro de esta clasificación, se seleccionaron aquellos que estaban relacionados con los riesgos de graves daños colectivos y riesgos de dańos individuales, respecto a los espectadores de los eventos deportivos que se celebren en la instalación, así como los referentes a los usuarios deportivos de las mismas, que se desglosan como se detalla a continuación (tabla 2):

Tabla 2. Riesgos de graves daños colectivos y daños individuales. (Gómez-Calvo, 2007).

\begin{tabular}{|c|c|}
\hline $\begin{array}{l}\text { Riesgos de } \\
\text { graves daños } \\
\text { colectivos }\end{array}$ & $\begin{array}{l}\text { 1. Incendio. } \\
\text { 2. Explosión de gas. } \\
\text { 3. Derrumbe o desplome. } \\
\text { 4. Avalancha } \\
\text { 5. Estampida. } \\
\text { 6. Atentado o amenaza del mismo. } \\
\text { 7. Grave accidente medio ambiental. } \\
\text { 8. Fenómenos de la Naturaleza extremos: tormen- } \\
\text { ta o lluvia torrencial, viento huracanado, terremo- } \\
\text { to, inundación, etc. }\end{array}$ \\
\hline $\begin{array}{l}\text { Riesgos } \\
\text { de daños } \\
\text { individuales }\end{array}$ & $\begin{array}{l}\text { 1. Accidentes debidos a la falta de adecuación, } \\
\text { condiciones y estado del recinto, las instalaciones } \\
\text { y el equipamiento. } \\
\text { 2. Accidentes debidos a la caída o desprendimien- } \\
\text { to de elementos u objeto en altura. } \\
\text { 3. Accidentes por práctica inadecuada, debido a } \\
\text { desconocimiento, o falta de enseńanza, adiestra- } \\
\text { miento, información o seńalización. } \\
\text { 4. Accidentes por descuido (caídas en el mismo o } \\
\text { distinto plano). } \\
\text { 5. Accidentes por uso inadecuado. } \\
\text { 6. Accidentes por conductas imprudentes o teme- } \\
\text { rarias, propias o de terceros. } \\
\text { 7. Indisposiciones repentinas. }\end{array}$ \\
\hline
\end{tabular}

En cuanto a la selección de los ítems para la creación de la herramienta, éstos se elaboraron a partir de la legislación estatal actual, normativas NIDE y UNE-EN, así como manuales y recomendaciones de buenas prácticas en instalaciones deportivas respecto a la seguridad de las mismas (tabla 3). 
Tabla 3. Legislación, normativas y manuales de buenas prácticas utilizados para la elaboración de la herramienta.

\section{Legislación estatal deportiva}

- Ley 10/1990, de 15 de octubre, del Deporte.

- Ley 19/2007, de 11 de julio, contra la violencia, el racismo, la xenofobia y la intolerancia en el deporte.

- Real Decreto 2816/1982, de 27 de agosto, por el que se aprueba el Reglamento General de Policía de Espectáculos Públicos y Actividades Recreativas.

- Real Decreto 1942/1993, de 5 de noviembre, por el que se aprueba el Reglamento de Instalaciones de Protección contra Incendios.

- Real Decreto 842/2002, de 2 de agosto, por el que se aprueba el Reglamento electrotécnico para baja tensión.

- Real Decreto 865/2003, de 4 de julio, por el que se establecen los criterios higiénico-Sanitarios para la prevención y control de la legionelosis.

- Real Decreto 314/2006, de 17 de marzo, por el que se aprueba el Código Técnico de la Edificación.

- Real Decreto 393/2007, de 23 de marzo, por el que se aprueba la Norma Básica de Autoprotección de los centros, establecimientos y dependencias dedicados a actividades que puedan dar origen a situaciones de emergencia.

- Real Decreto 1027/2007, de 20 de julio, por el que se aprueba el Reglamento de Instalaciones Térmicas en los Edificios.

- Real Decreto 203/2010, de 26 de febrero, por el que se aprueba el Reglamento de Prevención de la Violencia, el racismo, la xenofobia y la intolerancia en el deporte.

\section{Normas NIDE y UNE.}

UNE-EN 1838:2000. Iluminación. Alumbrado de emergencia.

UNE-EN 12193:2000. Iluminación. Iluminación de instalaciones deportivas.

- UNE-EN 749:2004/AC:2006. Equipos de campos de juego. Porterías de balonmano. Requisitos de seguridad y funcionales, métodos de ensayo.

- UNE-EN 1271:2004/AC:2006. Equipamiento de los campos de juego. Equipos de voleibol. Requisitos funcionales y de seguridad, métodos de ensayo.

- UNE-EN 1509:2005. Equipos de campos de juego. Equipos de bádminton. Requisitos funcionales y de seguridad, métodos de ensayo.

- UNE-EN 1270:2006. Equipos de campos de juego. Equipos de baloncesto. Requisitos funcionales y de seguridad. Métodos de ensayo.

- UNE-EN 14904:2007. Superficies para áreas deportivas. Especificaciones para suelos multi deportivos de interior.

UNE-EN 13200-4:2008. Instalaciones para espectadores. Parte 4: Asientos. Características de producto.

NIDE 1: Normas de Proyecto Campos Pequeños; Salas y Pabellones.

- NIDE 1: Normas Reglamentarias Campos Pequeños: Baloncesto, Balonmano, Hockey, Bádminton y Fútbol Sala.

\section{Manuales de Buenas Prácticas}

Buenas prácticas en Instalaciones Deportivas. Consejo Superior de Deportes, 2009.

Guía para el autocontrol de la seguridad en instalaciones deportivas. Instituto de Biomecánica de Valencia, 2009.

Seguridad en Instalaciones Deportivas. Consejo Superior de Deportes, 2010.

Legislación y Documentos Técnicos de Referencia en Instalaciones Deportivas. Consejo Superior de Deportes, 2010.

Los 218 ítems que conforman el checklist se dividieron en las siguientes áreas (tabla 4):

Tabla 4. Áreas de división del cuestionario de validación.

\begin{tabular}{clcc}
\hline Área & & No de ítems & \% del total \\
\hline 1. & Exteriores y acceso. & 20 & 9,17 \\
2. & Recepción y control. & 11 & 5,04 \\
3. & Deambulación (excepto graderíos). & 30 & 13,76 \\
4. & Deambulación hacia plantas superiores. & 17 & 7,79 \\
5. Graderíos. & 16 & 7,33 \\
\hline 6. & Aseos y vestuarios. & 28 & 12,84 \\
7. & Espacio deportivo. & 47 & 21,55 \\
8. Emergencia. & 29 & 13,30 \\
9. Evacuación. & 11 & 5,04 \\
10. Mantenimiento y Planes de Prevención y Actuación. & 9 & 4,12 \\
TOTAL & 218 & 100 \\
\hline
\end{tabular}




\section{Procedimiento de construcción de la herramienta}

Para elaborar la herramienta, se realizó inicialmente una minuciosa revisión de la legislación estatal actual, normativa no obligatoria, manuales de buenas prácticas y artículos referentes a la seguridad en instalaciones deportivas, por parte de los investigadores participantes en el presente estudio.

Una vez seleccionada la literatura relacionada con el objeto de la investigación, se procedieron a extraer los ítems atendiendo al criterio de prioridad para la inclusión de los mismos en su obligatoriedad, siendo la legislación en vigor incluida en primer lugar y, dentro de ésta en caso de ambigüedad, se atendió a la opción más restrictiva del riesgo presentado. Posteriormente se añadieron aquellos normativos no obligatorios pero claramente recomendados siguiendo a Durá (2000), el cual destaca que se hace necesario por tanto que la normativa existente, que actualmente no es de obligado cumplimiento, cobre este reconocimiento.

La distribución de los ítems seleccionados previamente, se llevó a cabo siguiendo las recomendaciones aportadas por el panel de expertos:

- División del cuestionario por zonas de la instalación para facilitar el trabajo al observador.

- Numeración de los ítems en función del área a supervisar para poder hacer referencia a ellos de forma sencilla y rápida.

- Agrupación de los planes en un apartado independiente.

- Creación de un área relativa a "emergencias" y otra relativa a "evacuación".

De estas modificaciones resultó la siguiente estructura:

- Exteriores y acceso.

- Recepción y control.

- Deambulación (excepto graderíos).

- Deambulación hacia plantas superiores.

- Graderíos.

- Aseos y vestuarios.

- Espacio deportivo.

- Emergencia.

- Evacuación.

- Mantenimiento y Planes de Prevención y Actuación.

\section{Validación de la herramienta}

Para comprobar la validez de contenido, la herramienta inicial creada se revisó por parte de cinco doctores en Ciencias de la Actividad Física y el Deporte de cuatro universidades españolas, así como por parte de un experto en seguridad en instalaciones deportivas a nivel nacional e internacional.

La validez de constructo se llevó a cabo a través de la realización de una prueba piloto en un gran pabellón de carácter municipal. Tras esta prueba, se reformularon tres ítems para facilitar su comprensión al auditor, se añadieron dos nuevos con motivo de la división de otros existentes, se eliminaron diez por considerarse irrelevantes y/o incluidos en otros anteriores y se cambiaron de área cuatro para facilitar el proceso de auditoría.

La recogida de datos se realizó por parte de dos investigadores de forma independiente a través de una triangulación intra-investigador, obteniendo una diferencia de 7 ítems, lo cual supone un 3,21\% del total. Estas discrepancias se basaron en tres de los casos a la interpretación de lo que se considera "al alcance del usuario", pudiendo éste o no ayudarse de implementos para llegar al factor de riesgo. Los cuatro ítems restantes estaban referidos a elementos estructurales tales como grietas y fisuras, óxido, anclajes y curvaturas, si bien la función del gestor en este aspecto es la comunicación al especialista responsable para su valoración y subsanación.

Procedimiento de la investigación

La investigación se realizó a través de un procedimiento de observación por parte de los investigadores, excepto en el área de "Mantenimiento y Planes de Prevención y Actuación" y el ítem referido a la formación de los trabajadores, donde se necesitó de la colaboración del gestor de la instalación. Ésta se llevo a cabo siguiendo la estructura mostrada en cinco fases (figura 1):

Figura 1. Cronología de la investigación.

$$
\text { Fase 1: Solicitud de autorización para la realización del estudio }
$$

$$
\text { Fase 2: Auditoria 1(estudio piloto) }
$$$$
\text { Fase 3: Auditoria } 2 \text { (estudio de la instalación) }
$$

$$
\text { Fase 4: Registro de los resultados }
$$

\section{Fase 5: Análisis y valoración}

Fase 1; solicitud de autorización para la realización del estudio. Se solicitó autorización previa al Concejal Delegado de Deportes del municipio a través de una carta en la cual se explicó de forma detallada el objetivo del estudio, su interés y motivación, así como el procedimiento que se iba a llevar a cabo. Una vez se aceptó la solicitud y se nombró a la persona responsable para supervisar la misma, se procedió a concertar la cita y a contactar con los gestores de referencia dados por la persona de apoyo, para la resolución de los ítems referidos al área de "Mantenimiento y Planes de Prevención y Actuación", 
así como otras dudas que pudieran surgir con motivo del desconocimiento de la instalación.

Fase 2; auditoría 1 (estudio piloto). Se procedió a la realización del estudio piloto a través del cuestionario provisional que ya ha había superado la validez de contenido. Éste se llevó a cabo en el mes de noviembre de 2014 por parte de dos investigadores participantes en el estudio, a través de cuyos resultados se modificó la herramienta inicial.

Fase 3; auditoría 2 (estudio de la instalación). Se realizó el estudio de la instalación con la lista de control definitiva tras la validación de constructo. Éste se desarrolló durante el mes de noviembre de 2014, a lo largo de dos jornadas consecutivas de 2 horas, ambas durante el horario de apertura habitual en horario de mañana, para interferir lo menos posible en el desarrollo de las actividades de mayor afluencia. Durante el proceso, los auditores estuvieron acompańados en todo momento por un trabajador de la Concejalía de Deportes designado por la institución, que cumplía las funciones de guía y resolución de posibles dudas, sin interferir en ningún momento en la toma de datos.

Fase 4; registro de los resultados. El registro de los resultados se realizó de forma inmediata en Microsoft Office Excel
2007, a través de una codificación binaria (1: cumple / 0: no cumple). En el caso de que no se presentara alguno de los ítems en la instalación, siempre y cuando esto no supusiera la inexistencia de riesgo descrito, sino de ausencia de esa área o material, éste se marcó como "cumple”, ya que no se presentaba el posible riesgo.

Fase 5; análisis y valoración. Una vez obtenidos los resultados, se procedió a su análisis y valoración a través de Microsoft Office Excel 2007 en base a:

Análisis por área: porcentaje de cumplimiento e incumplimiento de los ítems incluidos respecto al total del área.

Análisis global: porcentaje de cumplimiento e incumplimiento global del cuestionario respecto al total.

\section{Resultados}

De la valoración de los riesgos de la instalación a través de la herramienta creada, se extrajo que el porcentaje de cumplimiento general se establece en un $82,11 \%$.

A continuación se exponen los ítems correspondientes a cada área, así como su cumplimiento u omisión (tabla 5):

Tabla 5. Cumplimiento del cuestionario respecto a la división por áreas.

\begin{tabular}{|c|c|c|c|c|}
\hline Área & Ítems totales & Cumplen & No cumplen & $\%$ cumplimiento \\
\hline Exteriores y Acceso & 20 & 19 & 1 & $95 \%$ \\
\hline Recepción y Control & 11 & 8 & 3 & $72,7 \%$ \\
\hline Deambulación (excepto graderíos) & 30 & 24 & 6 & $80 \%$ \\
\hline Deambulación hacia Plantas Superiores & 17 & 15 & 2 & $88,2 \%$ \\
\hline Graderíos & 16 & 14 & 2 & $87,5 \%$ \\
\hline Aseos y Vestuarios & 28 & 23 & 5 & $82,1 \%$ \\
\hline Espacio Deportivo & 47 & 40 & 7 & $85,1 \%$ \\
\hline Emergencia & 29 & 19 & 10 & $65,5 \%$ \\
\hline Evacuación & 11 & 10 & 1 & $90,9 \%$ \\
\hline Mantenimiento y Planes de Prevención y Actuación & 9 & 7 & 2 & $77,7 \%$ \\
\hline TOTAL & 218 & 179 & 39 & $82,11 \%$ \\
\hline
\end{tabular}

Área 1 (Exteriores y accesos): la instalación no dispone de cámaras de video vigilancia y los responsables de la instalación comunican que no disponen de ellas en ninguna zona del pabellón, por lo que se incumple en todas las áreas en las que se ha tenido en cuenta.

Área 2 (Recepción y control): no se expone el Reglamento de Régimen Interno en lugar visible y se observa que la instalación no dispone de Unidad de Control Organizativo.

Área 3 (Deambulación excepto graderíos): se detectan deficiencias en el pavimento en dos aspectos: malas condiciones antideslizantes y zona con hundimientos. Se observan asimismo papeleras de dimensiones muy superiores al máxi- mo permitido en la zona. Se encuentra material deportivo y de oficina que puede llegar a obstaculizar el paso. Se presentan partes activas de la instalación eléctrica al alcance de los usuarios y espectadores (cableado en mal estado sin sujeción) y sin la protección adecuada.

Área 4 (Deambulación hacia plantas superiores): se advierte el incumplimiento de dos ítems referentes a las escaleras para el acceso a las mismas. Concretamente, debido a la antigüedad de la instalación, algunas de ellas disponen de bocel en sus peldaños, en contra de lo que marca el Documento Básico de Seguridad de Utilización y Accesibilidad del Código Técnico de Edificación respecto a los recorridos de 
evacuación. Asimismo se detecta una ausencia de pasamanos en una de las escaleras, incumpliendo nuevamente el documento mencionado anteriormente.

Área 5 (Graderíos): se han detectado dos incumplimientos: uno referido a las salidas de los mismos ya que, si bien existen algunas salidas que cumplen con el ancho estipulado, éstas son insuficientes respecto a lo que establece el Reglamento General de Policía de Espectáculos Públicos y Actividades Recreativas atendiendo al aforo de la instalación. Por otro lado, se detecta que el número de asientos por fila es superior a los 18 establecidos como máximo por el Reglamento anterior, encontrando filas con hasta treinta localidades, suponiendo un riesgo de carácter colectivo de accidente en condiciones de evacuación y emergencia.

Área 6 (Aseos y vestuarios): se detectan zonas de penumbra en algunas de las duchas de los extremos, al sólo disponer de una luminaria para todas ellas y quedar zonas sin iluminación directa. También se ha advertido que existen partes activas de la instalación eléctrica al alcance del usuario sin ningún tipo de protección y junto a zonas húmedas, como son los lavabos. Los conductos de agua, si bien cumplen con algunos de los requisitos marcados, éstos se encuentran a la vista y alcance de los usuarios, por lo que pueden ser manipulados. Respecto a los pulsadores temporizados en las duchas, sólo están instalados en algunas de ellas. Referente a la posible asistencia a personas con movilidad reducida, la instalación no contempla ningún mecanismo de solicitud de ayuda, por lo que ante situaciones de indisposiciones repentinas no hay ningún canal de comunicación entre el usuario y el personal de la instalación.

Área 7 (Espacio deportivo): se observa que los paramentos verticales de la instalación no disponen de sistema de amortiguación. Respecto a los espacios de seguridad para la práctica deportiva, se localiza material deportivo e institucional sobre estas franjas durante el desarrollo de las actividades, por lo que la función para la cual han sido diseñados no se cumple. Ninguno de los equipamientos deportivos evaluados lleva adheridas las recomendaciones de uso y consignas relativas a la seguridad. En cuanto a las redes de protección, si bien están correctamente ancladas, éstas presentan roturas de pequeńa magnitud. Por último, la instalación carece de túneles de protección.

Área 8 (Emergencia): de los ítems incumplidos se extrae que la instalación no dispone de ciertos elementos de protección contra incendios como son detectores automáticos de incendios, rociadores, pulsadores, sistema de control de humos o sectores diferenciados para el control del incendio con puertas resistentes al fuego. Más llamativo es si cabe la ausencia de extintores cada quince metros como establece la legislación vigente en toda la zona de deambulación de espectadores y usuarios, encontrando únicamente en la zona de oficinas y salas polivalentes. Respecto a la formación e información de usuarios y trabajadores, se detecta que no existe información expuesta en la instalación acerca de qué hacer en caso de emergencia por parte de los ocupantes de la misma. En cuanto a la formación en seguridad impartida a los trabajadores, si bien recibieron en su día en relación a la actuación en caso de emergencia y utilización de medios de protección manual, ésta no les fue impartida a todos y se considera desactualizada debido al tiempo transcurrido desde que se realizó. Relativo a la comunicación en caso de emergencia, la instalación dispone de megafonía pero ésta no cubre toda su extensión y no dispone de señales visuales y acústicas. Se detecta un incumplimiento relativo a la iluminación de emergencia en el caso de las escaleras, al no disponer de pilotos de señalización en sus escalones.

Área 9 (Evacuación): en esta área sólo se ha encontrado el incumplimiento de uno de los ítems, al encontrarse algunas salidas de evacuación cerradas con candados durante el horario de apertura de la instalación en las plantas altas, si bien aquellas pertenecientes a las plantas bajas estaban habilitadas para una evacuación inminente.

Área 10 (Mantenimiento y planes de prevención y actuación): se detecta que no se dispone de ningún director ni responsable de seguridad al quedar vacante ese puesto y estar pendiente de posible reasignación. Esto conlleva que los aspectos analizados en el presente trabajo, no son competencia directa de ninguno de los trabajadores y eso dificulta su evaluación, subsanación y control. Al igual que pasaba en el área de emergencias con la formación respecto al personal, aquella relativa a las condiciones de evacuación debe ser realizada por todos ellos y actualizada.

\section{Discusión.}

La herramienta creada ha permitido detectar y evaluar los riesgos presentes en la instalación deportiva, consiguiendo con los resultados obtenidos disponer de la información necesaria para cumplir con la máxima de la prevención.

El resultado de cumplimiento obtenido, que supone el $82,11 \%$, si bien es aceptable entendemos que toda desviación del $100 \%$ supone un riesgo a valorar que debe de ser contemplado y subsanado en la medida de lo posible.

Respecto a la naturaleza de los ítems, al haber incluido en su desarrollo tanto los requisitos establecidos legalmente, como aquellos que tienen carácter normativo o que se consideran recomendables, la gravedad del incumplimiento de cada uno de los puntos, depende de este hecho y de las posibles consecuencias derivadas de la no subsanación del riesgo.

El área de "Exteriores y accesos" es aquella que consigue un porcentaje mayor de cumplimiento alzándose con un 95\%, siendo la ausencia de cámaras de video vigilancia el único ítem que incumple. Éste no supone un elemento obligatorio, aunque es recomendable para la detección de posibles situaciones 
que impliquen un riesgo en los aledaños de la instalación, así como en el control de accesos, ya que su inclusión permitiría detectar una situación crítica en el mismo momento en el que el problema ocurre (Praditsathaporn, Kaviya, y Yupapin, 2010).

Dentro de la zona de "Recepción y control" no se expone el Reglamento de Régimen Interno, lo cual puede derivar en un aumento de los riesgos individuales produciéndose accidentes por práctica inadecuada, debido a desconocimiento, falta de enseñanza, adiestramiento, información o señalización (Gómez-Calvo, 2007). El riesgo puede ser disminuido al informar a los usuarios de la normativa relacionada con la seguridad y los riesgos asociados a su participación (McGregor y MacDonald, 2000).La instalación no dispone de Unidad de Control Organizativo al no haberle sido requerida por las instituciones competentes con anterioridad, aunque al haber albergado eventos de carácter internacional y otras competiciones nacionales consideradas de riesgo, su instalación debería de haber sido prevista. Además del aspecto de control, estos medios tienen un fuerte poder disuasorio, por lo que una buena gestión de los mismos puede evitar comportamientos inadecuados por parte de los aficionados (Giulianotti y Klauser, 2010). Asimismo, un elemento fundamental del control de masas es una adecuada comunicación entre trabajadores, asistentes y entre la dirección y los asistentes (Abbott y Geddie, 2000).

Si atendemos a los ítems referidos a las áreas de "Deambulación”, aquellos que se incumplen están relacionados con accidentes de tipo individual del Código Técnico de Edificación (CTE), si bien se debería atender especialmente al material deportivo y de oficina que obstaculiza el paso, ya que algunos se encuentran en recorridos de vías de evacuación. El área correspondiente a "Aseos y vestuarios", se caracteriza también por el incumplimiento de cinco puntos, atendiendo al igual que en el caso descrito anteriormente, a riesgos de tipo individual. Los vestuarios, duchas y pavimentos entre otros, son las zonas de mayor riesgo para los usuarios, sobre todo para discapacitados, que deben de ser contempladas especialmente en los protocolos del riesgo (Gallardo, García-Tascón y Burillo, 2008).

Los "Graderios", si bien presentan un porcentaje de cumplimiento alto (87,5\%), muestran dos deficiencias que pueden tener consecuencias importantes: salidas insuficientes y un exceso de localidades por fila. Estos puntos, relacionados con el control de masas, requieren de prioridad para su subsanación al constituir uno de los aspectos clave en la gestión de la seguridad (Abbott y Geddie, 2000).

Dentro del "Espacio deportivo" propiamente dicho, una medida preventiva recomendable sería la colocación de amortiguación en los paramentos verticales que, si bien no es obligatorio, disminuye el riesgo de accidente por traumatismo, cuya mortalidad respecto al total de los producidos en el ámbito deportivo se sitúa entre el 20-25\% (Carré, 2014). Basándonos nuevamente en la prevención, destaca que los equipamientos deportivos analizados no disponen de las recomendaciones de uso y consignas de seguridad adheridos a los mismos, lo cual puede desencadenar un accidente por el desconocimiento del usuario, aunque el equipamiento cumpla con las medidas de seguridad requeridas. En los grandes eventos deportivos, las diferencias entre los participantes y espectadores del mismo, pueden ocasionar conflictos que deriven en problemas muchos más complejos (Shangjun y Xinjian, 2012), por lo que la instalación de túneles de protección durante la celebración de los mismos debe de ser contemplada.

De los resultados obtenidos, el área más sensible y con un grado de cumplimiento más bajo (65,5\%) es la de "Emergencias", sobre todo en los ítems referidos a incendios, lo cual debería suponer la realización de una serie de actuaciones urgentes debido a la gravedad de los mismos. Otro de los factores a destacar es el relativo a la información, ya que no se exponen al usuario pautas sobre qué hacer en caso de emergencia, y los sistemas de comunicación presentan carencias importantes. Los incumplimientos detectados se pueden ver acentuados en la realización de eventos deportivos, debido a la importancia que las medidas de gestión de emergencias tienen en este ámbito (Junying, 2012).

Por último destacar la importancia de realizar y renovar la formación proporcionada a los trabajadores de la instalación deportiva, ya que la relativa a "Emergencias" y "Evacuación”, o no se ha realizado o está obsoleta, siendo no sólo los responsables y titulares de la instalación los encargados de velar por la seguridad de los usuarios y espectadores, sino también los trabajadores de las instalaciones deportivas objeto de estudio. Esta formación en la evaluación y análisis de las amenazas, es necesaria para asegurar una correcta respuesta e intervención frente un incidente (Hall, Marciani, Cooper y Phillips, 2010), necesitando asimismo de un buen conocimiento del contexto en el cual van a implementar sus programas de seguridad, para poder hacerlo adecuadamente (Christoffel y Gallagher, 2006; McGregor y MacDonald, 2000).

\section{Conclusiones.}

La utilización de la herramienta creada, ha puesto de manifiesto la importancia que tiene el control de los riesgos que se presentan en la instalación ya que, si bien el porcentaje de incumplimiento total es del 17,88 \%, algunos de ellos eran de carácter grave contraviniendo lo marcado legalmente y pudiendo desencadenar una serie de accidentes con fatales consecuencias.

Es por ello que se debe incidir en la necesidad de atender a la prevención como máxima en seguridad, por parte de titulares de las instalaciones y gestores deportivos, garantizando a espectadores, usuarios y trabajadores un óptimo control de los riesgos que pudieran presentarse. 
Algunas de las limitaciones que se pueden presentar en la aplicación de la presente herramienta son las siguientes:

- En el caso de investigaciones realizadas por individuos ajenos a la entidad, la información relativa a los planes y formación, puede ser transferida de forma errónea al prever por parte de la dirección de la instalación un resultado negativo de cumplimiento.

- Para la comprensión de algunos de los ítems, se requiere un conocimiento de estos puntos por parte del gestor deportivo o, en su defecto, de contar con un equipo de apoyo multidisciplinar para la resolución de dudas al respecto.

- La herramienta debe de ser actualizada si así lo hace la legislación y normativa en la que se ha basado su elaboración.
Como líneas futuras, sería interesante extender el estudio a varios grandes pabellones, así como ampliar esta investigación a otros tipos de riesgos como son los mencionados en el presente estudio o los antrópicos.

Con lo expuesto anteriormente, se ha buscado que la herramienta pueda ser utilizada por parte tanto de los investigadores en gestión deportiva para la realización de análisis de los riesgos presentes, como por parte de los gestores y responsables de las instalaciones deportivas señaladas, con la finalidad de que se convierta en una base para el control y valoración de los riesgos de grandes dańos colectivos e individuales que puedan presentarse en los grandes pabellones deportivos. Es por ello que los resultados que se obtengan, pretenden ser rigurosos, cuantificables y comparables.

\section{Referencias bibliográficas}

1. Abbott, J. L., \& Geddie, M. W. (2000). Event and venue management: Minimizing liability through effective crowd management techniques. Event Management, 6(4), 259-270.

2. Carré, F. (2014). La mort subite liée à la pratique sportive. La Presse Médicale, 43(7), 831-839.

3. Christoffel, T., \& Gallagher, S. (2006). Injury prevention and public health. Practical knowledge, skills, and strategies (2nd ed.). Sudbury: Jones and Bartlett Publishers.

4. De Castro, C. F., Simóes, D. C. M., Delamarque, E. V., \& Pepe, V. L. E. (2014). Eventos de massa, desastres e Saúde Pública. Revista Ciência \& Saúde Coletiva, 19(9), 3717-3730.

5. Durá, J. (2000). Situación del equipamiento deportivo instalado en la Comunidad de Murcia con relación a los criterios técnicos establecidos en las normativas técnicas europeas. Revista Biomecánica, 26, 17-20.

6. Finch, C., Donaldson, A., Mahoney, M., \& Otago, L. (2009). The safety policies and practices of community multi-purpose recreation facilities. Safety Science, 47, 1346-1350.

7. Fuller, C.W. (2007). Managing the risk of injury in sport. Clinical Journal of Sport Medicine, 17, 182-187.

8. Gallardo, L., García-Tascón, M., \& Burillo, P. (2008). New sports management software: A needs analysis by a panel of Spanish experts. International Journal of Information Management, 28(4), 235 245.

9. Giulianotti, R., \& Klauser, F. (2010). Security governance and sport mega-events: Toward an interdisciplinary research agenda. Journal of Sport \& Social Issues, 34(1), 49-61.

10. Gómez-Calvo, J.L. (2004). Deporte y Seguridad ¡Anatomía de dos gigantes!. Recuperado de http://www.belt.es/expertos/HOME2_experto. asp?id=2127

11. Gómez-Calvo, J.L. (2005). La gestión de la seguridad en las instalaciones y recintos deportivos. Recuperado de http://www.belt.es/expertos/ HOME2_experto.asp?id=2534.

12. Gómez-Calvo, J.L. (2007). La seguridad dentro de la organización de grandes eventos deportivos. Recuperado de http://www.belt.es/expertos/HOME2_experto.asp?id=3648

13. Gómez-Calvo, J.L. (2013). Seguridad de espectáculos y actividades re- creativas, deportivas, musicales y sociales. Recuperado de http://www. belt.es/expertos/HOME2_experto.asp?id=6780

14. Gómez-Calvo, J.L. (2014). Gestión de la seguridad presente y futura, en el ámbito recreativo y deportivo. Recuperado de http://www.belt.es/ expertos/HOME2_experto.asp?id=7071.

15. Hall, S., Cooper, W., Marciani, L. \& McGee, J. (2012). Security management for sports and special events: An interagency approach to creating safe facilities. Champaign: Human Kinetics.

16. Hall, S., Marciani, L., Cooper, W. \& Phillips, J. (2010). Needs, concerns, and future challenges in security management of ncaa division I football events: An intercollegiate facility management perspectives. Journal of Venue and Event Management, 1(2):1-16.

17. Junying, A. (2012). Emergency of Large Scale Engineering Sports Events. Systems Engineering Procedia, 4, 416-423.

18. López-Fernández, J., Sánchez-Sánchez, J., García-Unanue, J., Burillo, P., Gude, R., Gómez-Calvo, J. L.,... Gallardo, L. (2014). Diseño de una Herramienta Para Evaluar la Adecuación de los Estadios de Fútbol Espańoles a las Medidas de Seguridad Obligatorias. Kronos, 13(2).

19. McGregor, I., \& MacDonald, J. (2000). Risk management manual for sport and recreation organizations. Corvallis, OR: Shelton-Turnbull $\mathrm{Pr}$ inters, Published by NIRSA.

20. Parkkari, J., Kujala, U.M., \& Kannus, P. (2001). Is it possible to prevent sports injuries? Review of controlled clinical trials and recommendations for future work. Sports Medicine 31, 985-995.

21. Parkkari, J., Kannus, P., Natri, A., Lapinleimu, I., Palvanen, M., Heiskanen, ...Jarvinen, M. (2004). Active living and injury risk. International Journal of Sports Medicine, 25, 209-216.

22. Praditsathaporn, S., Kaviya, S., \& Yupapin, P. P. (2010). Sport safety improvement using security camera without audience privacy violation. Procedia-Social and Behavioral Sciences, 2(1), 62-67.

23. Requa, R., DeAvilla, L., \& Garrick, J. (1993). Injuries in recreational adult fitness activities. American Journal of Sports Medicine, 21, 461467.

24. Shangjun, W. \& Xinjian, Z. (2012). Problems of Mega-events Engineering Risk Management in China. Systems Engineering Procedia, 4 431-437. 